Check for updates

Cite this: Chem. Commun., 2017, 53, 11008

Received 9th August 2017. Accepted 23rd August 2017

DOI: $10.1039 / \mathrm{c} 7 \mathrm{cc} 06211 \mathrm{~g}$

rsc.li/chemcomm

\section{A one-pot radioiodination of aryl amines via stable diazonium salts: preparation of ${ }^{125}$ I-imaging agents $\dagger$}

\author{
Nikki L. Sloan, ${ }^{a}$ Sajinder K. Luthra, ${ }^{b}$ Graeme McRobbie, ${ }^{b}$ Sally L. Pimlott ${ }^{c}$ and \\ Andrew Sutherland (D) *a
}

\begin{abstract}
An operationally simple, one-pot, two-step tandem procedure that allows the incorporation of radioactive iodine into aryl amines via stable diazonium salts is described. The mild conditions are tolerant of various functional groups and substitution patterns, allowing latestage, rapid access to a wide range of ${ }^{125}$-labelled aryl compounds and SPECT radiotracers.
\end{abstract}

The combination of radionuclide labelled molecules and nuclear imaging techniques has become important for the study of dynamic biochemical processes at the molecular and cellular levels. ${ }^{1}$ The application of these technologies has also extended into many aspects of medicine and healthcare and include the diagnosis and prognosis of disease, as well as utilisation in the drug discovery process, such as understanding drug action and establishing treatment strategies. ${ }^{2}$ A key technique within this field is single-photon emission computed tomography (SPECT) which, in combination with radioactive tracers, is used for both preclinical in vitro measurements $\left({ }^{123} \mathrm{I} /{ }^{125} \mathrm{I}\right)$ and in vivo diagnostic imaging $\left({ }^{123} \mathrm{I}\right) .^{3}$

Despite the importance of SPECT imaging, one of the limitations preventing more widespread application of this technique is the dearth of methods for incorporating radioiodine into small molecules. In general, SPECT tracers bearing an iodoarene unit are produced by a substitution reaction using an electrophilic source of radioactive iodine, prepared under oxidising conditions. ${ }^{3 a}$ The most commonly used approach involves the conversion of aryl halides into the corresponding organostannane via palladium(0) catalysis, followed by an oxidative iodo-destannylation reaction (Fig. 1a). ${ }^{2,3 a}$ While this method generates SPECT imaging agents

\footnotetext{
${ }^{a}$ WestCHEM, School of Chemistry, The Joseph Black Building, University of Glasgow, Glasgow G12 8QQ, UK. E-mail: Andrew.Sutherland@glasgow.ac.uk; Fax: +44 (0)141330 4888; Tel: +44 (0)141330 5936

${ }^{b}$ GE Healthcare, The Grove Centre, White Lion Road, Amersham, HP7 9LL, UK

${ }^{c}$ West of Scotland PET Centre, Greater Glasgow and Clyde NHS Trust,

Glasgow G12 OYN, UK

$\dagger$ Electronic supplementary information (ESI) available: Experimental procedures, spectroscopic data, radio-HPLC traces and NMR spectra for all compounds. See DOI: $10.1039 / \mathrm{c} 7 \mathrm{cc} 06211 \mathrm{~g}$
}

with high specific activity and radiochemical purity, ${ }^{4}$ there are obvious toxicity risks associated with the generation of clinical tracers from organotin compounds. In addition, the unstable nature of organotin compounds prevents long-term storage and can result in unreliable radioiodination. ${ }^{5}$ Although there are significant issues associated with this approach, there have been relatively few reports of new methods for the radioiodination of aryl compounds. ${ }^{6}$ In 2013, we reported a nickel(0)-catalysed radioiodination of aryl and heteroaryl bromides (Fig. 1b). ${ }^{7}$ While fast and efficient, this method requires elevated temperatures $\left(180{ }^{\circ} \mathrm{C}\right)$ for complete conversion. More recently, the groups of Gouverneur and Zhang described the copper-mediated radioiodination of aryl boronic acids and esters (Fig. 1c). ${ }^{8-11}$ Besides showing a broad substrate scope, these methods were used for the preparation of various SPECT imaging agents.,

We were interested in developing a new general method for the radioiodination of aryl compounds with the criteria of

a) Two-step radioiodo-destannylation approach

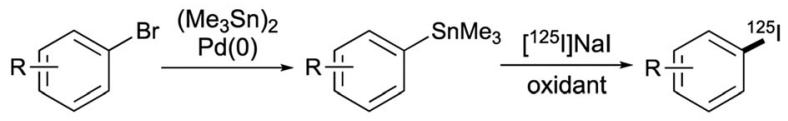

b) Nickel(0)-mediated radioiodination of aryl bromides

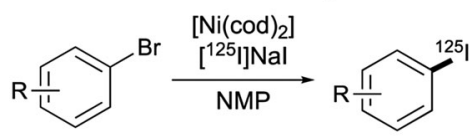

c) Copper-mediated radioiodination of aryl boronic acids and esters

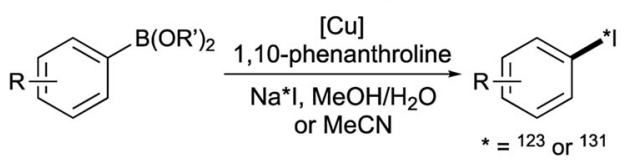

d) One-pot radioiodination of anilines- This work

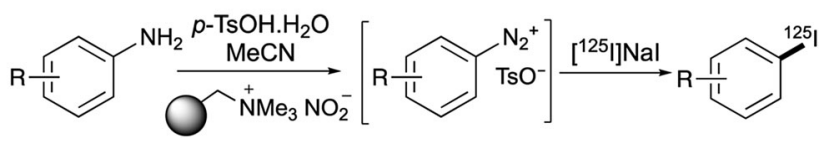

Fig. 1 Methods for radioiodination of arenes. 
using widely available starting materials and mild reaction conditions. The preparation of diazonium salts from aryl amines followed by a Sandmeyer-type reaction is a common approach for the preparation of aryl iodides. ${ }^{12}$ However, previous attempts to use this two-step transformation for radioiodination were largely abandoned. ${ }^{13}$ The harsh acidic conditions required for the formation of diazonium salts and their unstable, potentially explosive nature led to unwanted by-products and low radiochemical yields. Here we report an operationally simple, one-pot radioiodination of aryl amines that overcomes these previous limitations. Formation of the diazonium salts under mild conditions, using a polymer-supported nitrite reagent and $p$-toluenesulfonic acid ( $p$-TsOH) as the proton source, followed by direct radioiodination, has produced labelled products in high radiochemical yields (Fig. 1d). Application of this new strategy for the preparation of various SPECT imaging agents is also described.

A number of groups have shown that the key limitations associated with the standard conditions of diazonium salt formation, such as the use of strong acids and sodium nitrite, which can lead to the release of nitrogen oxides, can be avoided using a polymer-supported nitrite reagent. ${ }^{14}$ These are simply prepared by the ion exchange of tetraalkylammonium functionalised resins, such as Amberlyst A-26, using an aqueous solution of sodium nitrite. Filimonov and co-workers have combined the use of a polymer-supported nitrite with mild acidic conditions ( $p$-TsOH) and showed that the resulting tosylate diazonium salts were thermally stable and could be used for the stepwise preparation of aryl iodides under aqueous conditions. ${ }^{14 c, d}$ These advances served as a starting point for the development of our rapid, onepot tandem radioiodination of aryl amines.

Our initial studies focused on the development of a mild, one-pot diazotisation-iodination of aryl amines that would also be amenable for radioiodination. Preliminary optimisation studies with 4-nitroaniline (1a) showed that when using acetonitrile as the solvent, all the reagents (polymer-supported nitrite, $p$-TsOH and sodium iodide) could be added together, resulting in a fast and efficient tandem conversion to the corresponding iodide, 2a ( $86 \%$ yield). These conditions were then investigated for the radioiodination of 4-nitroaniline (1a), where the radiochemical yields (RCY) were determined by radio-HPLC analysis of the crude product. ${ }^{15}$ Using $\left[{ }^{125} \mathrm{I}\right] \mathrm{NaI}$ (4-6 MBq solution in water) as the limiting reagent and three equivalents of both the polymersupported nitrite reagent and $p$-TsOH gave a low RCY (Table 1, entry 1). The concentration of $\mathbf{1 a}$ in the reaction mixture was then varied and using more dilute conditions, which allowed better mixing of reagents, resulted in a dramatic increase in the RCY of $2 \mathrm{a}$. In particular, a concentration of $0.018 \mathrm{~mol} \mathrm{~L}^{-1}$ of $\mathbf{1 a}$ $(0.5 \mathrm{mg}$ of $1 \mathrm{a}$ in $200 \mu \mathrm{L}$ of solvent) gave an excellent RCY of $93 \%$ (entry 3 ). The corresponding radio-HPLC chromatogram for this reaction showed a particularly clean transformation with no other radiolabelled side-products (Fig. 2). Other variables of the one-pot tandem process were also investigated such as reaction temperature and the number of equivalents of reagents (entries 4-8). Higher temperatures led to a drop in the RCY due to the formation of 4-nitrophenol as a side-product, while higher quantities of reagents again led to inefficient mixing of the
Table 1 Optimisation of the one-pot diazotisation-radioiodination of 4nitroaniline $(\mathbf{1} \mathbf{a})^{a}$

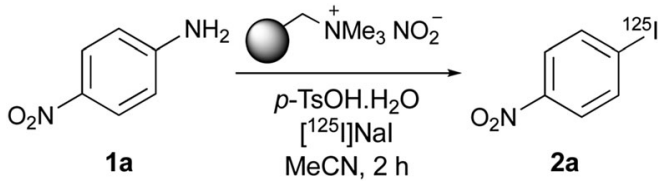

\begin{tabular}{|c|c|c|c|c|}
\hline Entry & Conc. of $1 \mathrm{a}\left(\mathrm{mol} \mathrm{L}^{-1}\right)$ & Eq. of reagents ${ }^{b}$ & Temp. $\left({ }^{\circ} \mathrm{C}\right)$ & RCY (\%) \\
\hline 1 & 0.18 & 3 & 20 & 4 \\
\hline 2 & 0.07 & 3 & 20 & 44 \\
\hline 3 & 0.018 & 3 & 20 & 93 \\
\hline 4 & 0.009 & 3 & 20 & 80 \\
\hline 5 & 0.018 & 3 & 40 & 65 \\
\hline 6 & 0.018 & 3 & 60 & 54 \\
\hline 7 & 0.018 & 1 & 20 & 82 \\
\hline 8 & 0.018 & 6 & 20 & 43 \\
\hline
\end{tabular}

${ }^{a}$ All reactions were performed using a $4-6 \mathrm{MBq}$ solution of $\left[{ }^{125} \mathrm{I}\right] \mathrm{NaI}$ in water $(0.01 \mathrm{~mL}) .{ }^{b}$ Polymer-supported nitrite and $p$-TsOH.

small-scale reaction and a lower RCY. Although a high RCY was achieved using one equivalent of both reagents (entry 7), the conditions used in entry 3 were deemed optimal for radioiodination.

The optimised radioiodination conditions were evaluated for the preparation of a range of ${ }^{125}$ I-labelled aryl compounds (Scheme 1). Under these standard conditions, electron-deficient and electron-rich compounds (1a-1h) with various substitution patterns were found to be compatible and converted to ${ }^{125}$ I-iodinated compounds in excellent RCYs. Only two substrates required further optimisation. The highest RCY of $97 \%$ for $4-\left[{ }^{125}\right.$ I] iodoanisole (2c) was achieved when the one-pot tandem process was conducted at $40{ }^{\circ} \mathrm{C}$, while a four-hour reaction gave the best RCY (94\%) for $2-\left[{ }^{125} \mathrm{I}\right]$ iodobenzophenone (2e).

Having demonstrated the general scope of the one-pot tandem diazotisation-radioiodination process on simple arenes, the next stage of this work was to show that it could also be applied to the synthesis of more complex biologically active aryl iodides and SPECT radiotracers (2i-2m, Scheme 1). ${ }^{16}$ Again, using $\left[{ }^{125} \mathrm{I}\right] \mathrm{NaI}$ as the limiting reagent under dilute conditions, it was found that an increase in the amount of both the polymersupported nitrite and $p$-TsOH (to six equivalents) was required to achieve good yields for a two-hour reaction. Further optimisation showed that a higher reaction temperature $\left(60^{\circ} \mathrm{C}\right)$ gave improved

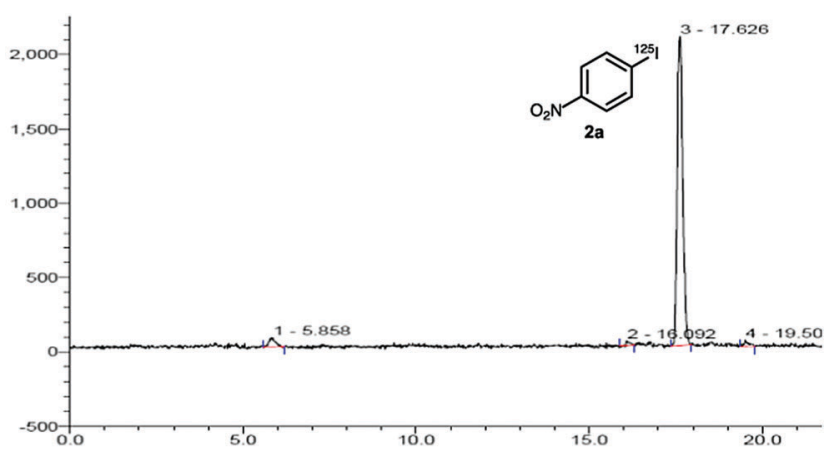

Fig. 2 Chromatogram obtained by analytical radio-HPLC of the reaction mixture from the radioiodination of 4 -nitroaniline (1a), showing a $93 \%$ yield of radioiodide incorporation. 


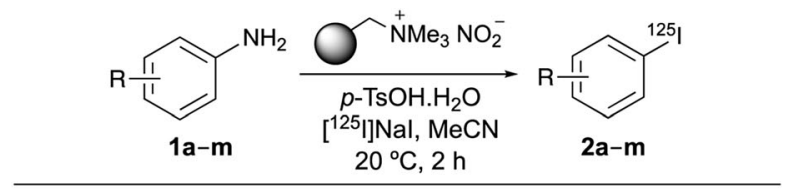<smiles>COc1ccc([Al]c2ccc(Br)cc2)cc1</smiles>
$\begin{array}{llll}\text { 2a, } 93 \% & \text { 2b, } 73 \% & \text { 2c, } 97 \%^{a} & \text { 2d, } 94 \%\end{array}$<smiles>COc1cc([123I])c([131I][Na])cc1OC</smiles>

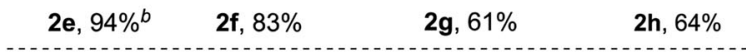

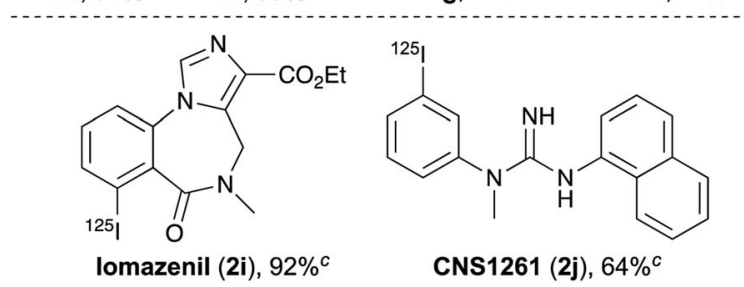<smiles>CN(C)c1ccc(-c2nc3ccc([Al])cc3o2)cc1</smiles><smiles>CCN(CC)C(=O)c1cc(-c2ccccc2[18F])c2ccccc2n1</smiles>

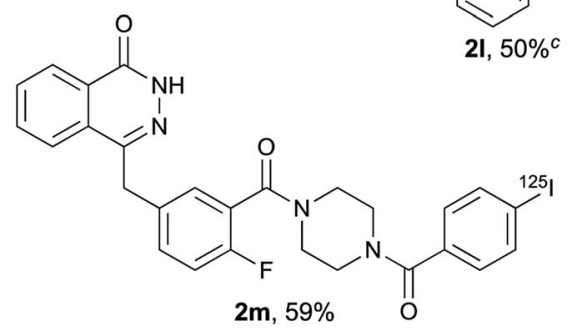

Scheme 1 Scope of one-pot diazotisation-radioiodination. ${ }^{a}$ Reaction was performed at $40{ }^{\circ} \mathrm{C}$. ${ }^{b}$ Reaction required $4 \mathrm{~h}$. ${ }^{c}$ Reaction was performed at $60^{\circ} \mathrm{C}$.

RCYs for some of the substrates. Overall, the one-pot transformation was effective for all the targets, leading to a new radiosynthesis of the well-established imaging agents iomazenil (2i) ${ }^{17}$ a SPECT tracer of central-type benzodiazepine receptors in brain tissue; CNS1261 (2j) ${ }^{18}$ a radioligand of the $N$-methyl-D-aspartate (NMDA) receptor; and IBOX (2k), a SPECT imaging agent of amyloid plagues. ${ }^{19}$ The best result was achieved for $\left[{ }^{125} \mathrm{I}\right]$ iomazenil (2i), with a particularly clean reaction and a RCY of $92 \%$. This is higher than that for the reported oxidative iodo-destannylation of a tri- $n$-butylstannane precursor $(70 \%)$, which was complicated by the formation of non-radioactive reduced and halogenated by-products. ${ }^{20}$ The synthesis of $\left[{ }^{125} \mathrm{I}\right]$ iomazenil (2i) was also found to be highly reproducible, with a RCY of $89 \pm 8 \%(n=9)$. This study has also allowed the first preparation of a radioiodinated version of quinoline-2-carboxamide 2l, a high-affinity agent $\left(K_{\mathrm{i}} 5 \mathrm{nM}\right)$ for the translocator protein (TSPO), a membrane protein that is

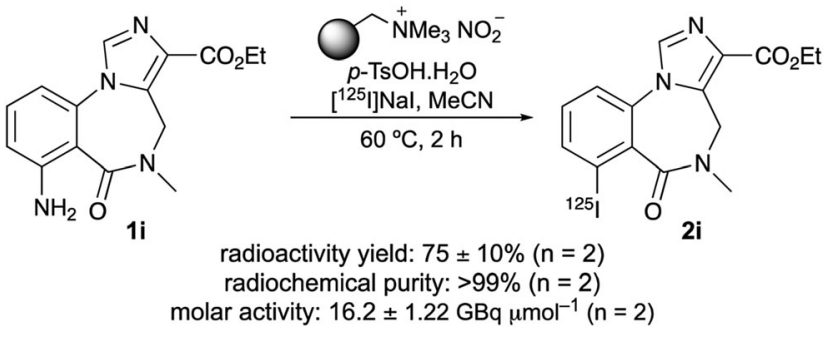

Scheme 2 Radiosynthesis of ${ }^{125}$ I]jiomazenil (2i).

overexpressed during chronic neurodegeneration or acute brain injury. ${ }^{21}$ As the radiosynthesis of this compound is now developed, the full potential of this tracer can be explored as a potential SPECT imaging agent of neuroinflammation processes associated with TSPO. The one-pot diazotisation-radioiodination was also effective for the preparation of phthalazinone $\mathbf{2 m}$, which is a nanomolar inhibitor ( $\mathrm{IC}_{50} 7 \mathrm{nM}$ ), and a SPECT imaging agent of poly(ADP-ribose) polymerase-1 (PARP-1), a therapeutic target for the treatment of cancer. ${ }^{22}$ The previous ${ }^{125}$ I-labelled synthesis of $\mathbf{2 m}$ was achieved by solid-state halogen exchange using a bromide precursor. Although this did give a 90\% RCY, a reaction temperature of $210{ }^{\circ} \mathrm{C}$ was required.

Besides using widely available starting materials, avoiding highly toxic reagents and harsh conditions, it should be emphasised that other advantages of this one-pot diazotisation-radioiodination process are a very simple work-up procedure and isolation protocol. Following completion of the reaction, the radioactive mixture is filtered to remove the polymer-supported nitrite, diluted and purified, directly by HPLC to yield the ${ }^{125}$ I-labelled compounds. These advantages were exemplified with the validation of the one-pot method for the synthesis and purification of $\left[{ }^{125} \mathrm{I}\right]$ iomazenil (2i). The amine precursor 1i was treated with $\left[{ }^{125} \mathrm{I}\right] \mathrm{NaI}(4-6 \mathrm{MBq})$, polymer-supported nitrite and $p$-TsOH at $60{ }^{\circ} \mathrm{C}$ for 2 hours (Scheme 2). After HPLC purification, $\left[{ }^{125} \mathrm{I}\right]$ iomazenil (2i) was isolated in $75 \pm 10 \%$ radioactivity yield. The radiochemical purity of $2 \mathbf{i}$ was measured to be $>99 \%$, with a molar activity of $16.2 \pm$ $1.22 \mathrm{GBq} \mu \mathrm{mol}^{-1}$. As with all reactions, identification of the product was confirmed using HPLC, demonstrating co-elution with a sample of unlabelled iomazenil.

In summary, an operationally simple, one-pot, two-step tandem process for the preparation of radioiodine containing arenes from readily available anilines has been developed. The use of mild conditions for diazonium salt formation resulted in a process with a broad scope that is tolerant of a wide range of functional groups and substitution patterns. In particular, this process allowed the preparation of a range of important SPECT imaging agents, including $\left[{ }^{125} \mathrm{I}\right]$ iomazenil, $\left[{ }^{125} \mathrm{I}\right] \mathrm{CNS} 1261$ and $\left[{ }^{125} \mathrm{I}\right] \mathrm{IBOX}$, as well as the first radiosynthesis of a high affinity agent of TSPO, which can now be used to study neuroinflammation. Current studies are investigating the application of this method for the development of novel SPECT imaging agents and the use of solid-phase extraction for more simple and rapid purification.

Financial support from Medical Research Scotland (studentship to N. L. S.), GE Healthcare Ltd and the University of Glasgow is gratefully acknowledged. 


\section{Conflicts of interest}

There are no conflicts to declare.

\section{Notes and references}

1 (a) S. M. Ametamey, M. Honer and P. A. Schubiger, Chem. Rev., 2008, 108, 1501; (b) P. W. Miller, N. J. Long, R. Vilar and A. D. Gee, Angew. Chem., Int. Ed., 2008, 47, 8998; (c) S. Preshlock, M. Tredwell and V. Gouverneur, Chem. Rev., 2016, 116, 719.

2 (a) M. J. Adam and D. S. Wilbur, Chem. Soc. Rev., 2005, 34, 153; (b) A. Signore, S. J. Mather, G. Piaggio, G. Malviya and R. A. Dierckx, Chem. Rev., 2010, 110, 3112; (c) S. L. Pimlott and A. Sutherland, Chem. Soc. Rev., 2011, 40, 149.

3 (a) R. H. Seevers and R. E. Counsell, Chem. Rev., 1982, 82, 575; (b) M. M. Khalil, J. L. Tremoleda, T. B. Bayomy and W. Gsell, Int. J. Mol. Imaging, 2011, 2011, 796025.

4 For example: S. L. Pimlott, L. Stevenson, D. J. Wyper and A. Sutherland, Nucl. Med. Biol., 2008, 35, 537.

5 For example: A. G. Horti, A. O. Koren, K. S. Lee, A. G. Mukhin, D. B. Vaupel, A. S. Kimes, M. Stratton and E. D. London, Nucl. Med. Biol, , 1999, 26, 175.

6 (a) N. L. Sloan and A. Sutherland, Synthesis, 2016, 2969; (b) D. T. Racys, S. A. I. Sharif, S. L. Pimlott and A. Sutherland, J. Org. Chem., 2016, 81, 772.

7 A. A. Cant, S. Champion, R. Bhalla, S. L. Pimlott and A. Sutherland, Angew. Chem., Int. Ed., 2013, 52, 7829.

8 T. C. Wilson, G. McSweeney, S. Preshlock, S. Verhoog, M. Tredwell, T. Cailly and V. Gouverneur, Chem. Commun., 2016, 52, 13277.

9 P. Zhang, R. Zhuang, Z. Guo, X. Su, X. Chen and X. Zhang, Chem. Eur. J., 2016, 22, 16783.

10 Radioiodination of boron derived arenes using chloramine-T has also been reported: (a) P. C. Srivastava, A. P. Callahan, E. B. Cunningham and F. F. Knapp, J. Med. Chem., 1983, 26, 742; (b) L. Yong, M.-L. Yao, J. F. Green, H. Kelly and G. W. Kabalka, Chem. Commun., 2010, 46, 2623.

11 A copper mediated non-radioactive iodination of aryl boronate esters using iodide as the limiting reagent has been reported: B. M. Partridge and J. F. Hartwig, Org. Lett., 2013, 15, 140.
12 (a) T. Sandmeyer, Ber. Dtsch. Chem. Ges., 1884, 17, 1633; (b) H. H. Hodgson, Chem. Rev., 1947, 40, 251; (c) C. Gali, Chem. Rev., 1988, 88, 765.

13 (a) G.-J. Meyer, K. Rössler and G. Stöcklin, J. Am. Chem. Soc., 1979, 101, 3121; (b) S. M. Moerlein, C. A. Mathis and Y. Yano, J. Labelled Compd. Radiopharm., 1986, 33, 1237; (c) D. S. Wilbur, Bioconjugate Chem., 1992, 3, 433.

14 (a) M. Caldarelli, I. R. Baxendale and S. V. Ley, Green Chem., 2000, 2, 43; (b) J. Merrington, M. James and M. Bradley, Chem. Commun., 2002, 140; (c) V. D. Filimonov, M. Trusova, P. Postnikov, E. A. Krasnokutskaya, Y. M. Lee, H. Y. Hwang, H. Kim and K.-W. Chi, Org. Lett., 2008, 10, 3961; (d) M. E. Trusova, E. A. Krasnokutskaya, P. S. Postnikov, Y. Choi, K.-W. Chi and V. D. Filimonov, Synthesis, 2011, 2154.

15 The radiochemistry nomenclature used in this paper is in accordance with the new guidelines recommended by the Society of Radiopharmaceutical Sciences. See: http://www.srsweb.org/nomenclature-guidelines and: P. H. Elsinga, EJNMMI Radiopharmacy and Chemistry, 2017, 2, 2.

16 See the ESI $\dagger$ for full details on the synthesis of each amine precursor.

17 For example: (a) S. S. Zoghbi, R. M. Baldwin, J. P. Seibyl, M. S. Al-Tikriti, Y. Zea-Ponce, M. Laruelle, E. H. Sybirska, S. W. Woods, A. W. Goddard, R. T. Malison, R. Zimmerman, D. S. Charney, E. O. Smith, P. B. Hoffer and R. B. Innis, Nucl. Med. Biol., 1992, 19, 881; (b) K. Mori, T. Mori, Y. Toda, E. Fujii, M. Miyazaki, M. Harada and S. Kagami, Brain Dev., $2012,34,478$.

18 (a) J. Owens, A. A. Tebbutt, A. L. McGregor, K. Kodama, S. S. Magar, M. E. Perlman, D. J. Robins, G. J. Durant and J. McCulloch, Nucl. Med. Biol., 2000, 27, 557; (b) R. J. J. Knol, K. De Bruin, B. L. F. Van Eck-Smit, S. Pimlott, D. J. Wyper and J. Booij, Synapse, 2009, 67, 557.

19 Z.-P. Zhuang, M.-P. Kung, C. Hou, K. Plössl, D. Skovronsky, T. L. Gur, J. Q. Trojanowski, V. M.-Y. Lee and H. F. Kung, Nucl. Med. Biol., 2001, 28, 887.

20 B. J. McBride, R. M. Baldwin, J. M. Kerr and J.-L. Wu, Appl. Radiat. Isot., 1991, 42, 173.

21 A. Blair, L. Stevenson, D. Dewar, S. L. Pimlott and A. Sutherland, MedChemComm, 2013, 4, 1461.

22 F. Zmuda, G. Malviya, A. Blair, M. Boyd, A. J. Chalmers, A. Sutherland and S. L. Pimlott, J. Med. Chem., 2015, 58, 8683. 